\title{
Hybrid Hand-off Scheme for Performance Improvisation of Wireless Networks
}

\author{
Prof. Vaishali Sarangpure \\ Assistant Professor, Department of Computer Science and Engineering, K.D.K. Women's College \\ India \\ vaishali.sarangpure@gmail.com
}

\begin{tabular}{|c|c|}
\hline Article History & Abstract \\
\hline $\begin{array}{l}\text { Article Submission } \\
29 \text { May } 2018 \\
\text { Revised Submission } \\
15 \text { July } 2018 \\
\text { Article Accepted } \\
29 \text { August } 2018 \\
\text { Article Published } \\
30 \text { September } 2018\end{array}$ & $\begin{array}{l}\text { Wireless systems administration is turning into an inexorably significant and well } \\
\text { known method of giving worldwide data access to clients progressing. Handoff, the } \\
\text { procedure where portable hubs select best passageway accessible to move } \\
\text { information and its postpones make a major issue. A great deal of research has been } \\
\text { done in most recent years to diminish the handoff delays happen in the various } \\
\text { degrees of WLAN correspondence. Because of the versatility of handoff, significant } \\
\text { viewpoint in WLAN and cell correspondences are substantially more significant. } \\
\text { Because of constrained scope of APs, WLAN likewise gives adequate data } \\
\text { transmission to continuous spilling administrations. In this research article, various } \\
\text { handoff plans are proposed to diminish the hand-off idleness and bolster quick hand- } \\
\text { off in IEEE 802.11 larcchitecture of wireless networks. In this article, we survey these } \\
\text { quick handoff plots subjectively. In this work it is to make accessible preparation for } \\
\text { prospect research on lessening the handoff inertness and give accentuation on } \\
\text { necessity of quick handover for consistent network. } \\
\text { Keywords: WLAN, Latency, Multiple radio scanning, Handoff process, packet loss }\end{array}$ \\
\hline
\end{tabular}

\section{Introduction}

The work of WLANs is tremendously favoured than wired systems consequently it is turning out to be basic part of the systems. WLAN utilizes high recurrence radio signs to transmit and get data over a separation. In physical and interface layers numerous conventions can be utilized to set up association in IEEE 802.11 and Wi-MAX. During the time spent handoff basically three substances are taking part to be specific station, earlier AP, and back AP. In IEEE 802.11 and cell frameworks hard handoff is utilized utilizing time division various access (TDMA) and recurrence division numerous entrance (FDMA). On the off chance that the old association is kept up until the new association is built up (make-before-break), it is known as delicate handoff [1][2].

As per the change in various remote systems, handoff can be ordered into two sections: Horizontal handoff and Vertical handoff. Level handoff is the intra-framework handoff like moving the progressing association starting with one AP then onto the next in various BSS in WLAN though in vertical handoff continuous association is moved between various remote systems or frameworks (between framework, for example, WLAN to cell or WLAN to GPRS frameworks and so on.

In WLAN architecture, the base station must rupture the association with earlier AP and join new access point, so during the hand-off time interim STA incapable to transmit or get any information. Various handoff strategies have been proposed on reducing the handoff dormancy or buffering information during this interim. Need to decrease handover inactivity increasingly more to utilize some ongoing applications, for example, VoIP, live communicating, numerous sorts of caution frameworks and video observation frameworks. Because of the constrained help scope of APs versatile STAs require as often as possible handoff [3][4]. 


\section{Existing techniques}

The related works away at different calculations to lessen the hand off idleness in remote system. Improving the IEEE 802.11 handoff to help mixed media traffic. The ideal system determination is key issue handoff and customary procedure of utilizing RSS isn't reasonable for incorporated situations of remote systems. In the 802.11 norm, a handoff strategy comprises of filtering, verification, and re-affiliation. In dynamic checking approach primarily to kill handoff inactivity by misusing the capability of numerous radios in WLAN gadgets. In this proposed Multi-Scan approach, requires neither changing the Access Points (APs), nor knowing about remote system topology hubs depend on utilizing their (conceivably inactive) second remote interface to entrepreneurially check and pre-partner with substitute APs and in the end consistently handoff progressing associations. The edge layout exchange occurs between the STA and the AP during the handoff technique. Hence, the handoff method causes some idleness, during which the STA can't send or get traffic [5].

The Handoff is the way toward moving the progressing meeting between versatile STA and comparing hub starting with one purpose of connection then onto the next purpose of connection or the equivalent. Because of the portability of gadgets, the handoff is a basic angle in WLAN and cell systems. Examining likewise called testing is the principal period of handoff procedure and it is the way toward choosing an appropriate AP from neighboring APs to handoff. The station acclimate the out of range status after different unanswered demands and starts the inquiry stage. Recommended another methodology in which STA begins search stage legitimately by barring the purpose behind disappointment of impacts on the grounds that above discovery strategy is long as appeared in and on the off chance that the chose AP via search stage is ebb and flow one, at that point handoff won't be executed [6].

Handoff process allows a customer to move around without meddling with the persistent relationship among STA and contrasting center point. It changes the present occupy in the current cell to another direct in the various cell or in the comparable. The time between time in the complete handoff process is known as handoff inaction. Handoff impacts the idea of organization genuinely [8][10]. Handoff occurs if the sign quality falls underneath a predefined edge level. The Quality-of organization (QoS) and cutoff of the framework may be impacted in light of handoff. There are a couple of essentials to diminish the horrible effect of handoff, for instance, handoff idleness must be as low as could be normal in light of the current situation, unquestionably the quantity of handoff should be immaterial [7][9].

\section{Proposed Methodology}

In WLAN handoff is the part of moving the ceaseless affiliation beginning with one AP then onto the following as a result of poor sign quality. Handoff is the path toward disassociating from the prior AP and setting up a relationship with the back AP. The need of handoff is perceived if signal quality (RSS) from current AP falls underneath the particular edge level. The L2 handoff of WLAN is hard-handoff and apportioned into three phases: sifting, confirmation, and re-association [16]. The complete handoff methodology can be assembled into two assorted savvy propels, (1) Discovery (checking), (2) Re-confirmation. An approval and re-relationship to the back AP are all things considered called Re-check. The handoff dormancy or delay is the aggregate of deferral gained by means of search and re-approval stage. The general handoff delay is the entire of the deferrals achieved by $\mathrm{m}$ solitary stages.

Scanning stage is the dreariest stage in the entire handoff process which is over $90 \%$ of the general handoff delay and the fundamental driver of MAC layer handoff inaction is looking at of available channels. Sifting can be described into two sorts: dynamic and inert. In detached inspecting each direct is tuned in by STA for the reference point diagrams for a fixed proportion of time. Channel is articulated unfilled and completed the checking of that channel. STA keeps things under control for Max Channel Time for extra responses from various APs on a comparable channel if an AP sends a response message.

Dynamic output diminishes the time taken to check yet builds the traffic load and furthermore expands power utilization. Utilizing channel checking best AP is chosen after this confirmation and re-affiliation stages start 
implies genuine handoff starts. For validation STA sends confirmation solicitation to the chose new AP and it sends the verification reaction to the STA, on the off chance that reaction isn't gotten by STA, at that point, STA couldn't re-partner to the new AP.

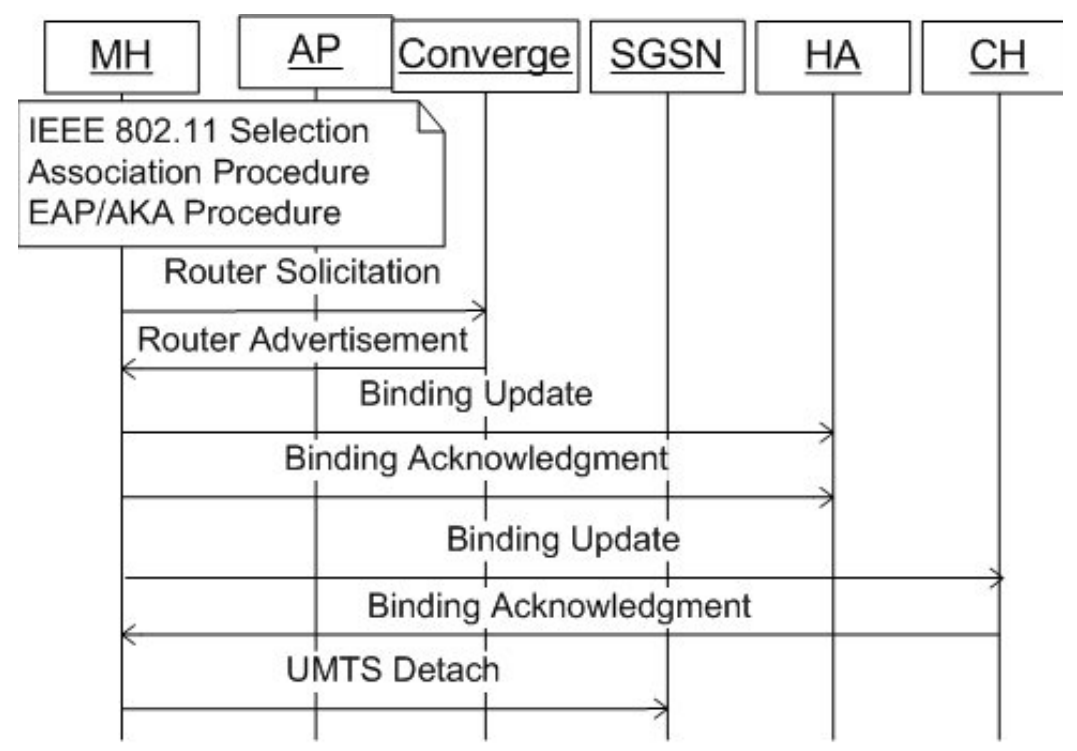

Fig 1:Basic structure of proposed hand off

To decrease the handoff, an effective MAC layer handoff convention is introduced. In case of Distributed Handoff conspire, to filter all the accessible channels, a portable hub chooses a few neighbouring hubs before it begins starting the MAC layer Handoff process. All channels are assembled and these gatherings are filtered by chosen neighbouring hubs independently. In every hub the quantity of checking channels is decreased and limited the filtering dormancy. To reduce the handoff latency in IEEE 802.11 based WN, a viable MAC layer handoff show is presented. If there should arise an occurrence of Distributed Handoff plot, to channel all the open channels, a flexible centre point picks a couple of neighbouring centres before it starts beginning the MAC layer Handoff process. An adaptable centre point requires centre points in a particular range to help it with analyzing the open channels when the handoff methodology is begun.

In this plan predefine the three RSS levels: RSSL, RSSH, RSSG Threshold to trigger handoff is RSSL By utilizing the associate hubs to frame a brief handoff bunch RSSH is utilized. Before beginning the handoff procedure by STA, it guarantee the presence of colleague hubs RSSH minimal higher than RSSL. To characterize the biggest good ways from the STA to its neighbour hubs RSSG is utilized. This plan comprise three principle parts, bunch development, dispersed output system and reserve conspire. At the point when the RSS is lower than RSSH, gathering process is activated in bunch development. STA examines neighbour hubs in run $r$ as the associate hubs; in this way, hubs N0, N1, N2, N3, and N4 are chosen in the given model.

AP information subject to the outcome of scattered yield segment can be taken care of in the store structure, since it is also serviceable for teammate centre points to re-band together with the proportional AP in a little while between time in view of closeness of the partner centre points to the STA. The partner centres will save the new AP in its holding structure with a lease time in the response of the discussed message with the new AP from STA to all colleague hubs. Just the latest AP is saved in the store, and the old AP is by and large overwritten. Exactly when the partner centre needs to begin handoff, in the essential it will endeavour to re-confirm with the AP set aside in the hold during the lease time. 


\section{Simulation Results}

Filtering stage is the most tedious stage, it has over $90 \%$ of the general handoff all out postponement. By utilizing the Pre-dynamic Scanning plan which works during typical availability, we can decrease the handoff defer time in location and search stage. In Pre-dynamic output STA start execution stage legitimately immediately in the location and search stage, it has bit of leeway of traffic load sharing and STA take choice to begin handover to new AP which is giving higher caliber than the past AP.

The Hexagonal cell idea is utilized to quicken the handoff procedure. The situation of versatile hub is acquired by utilizing GPS or some other restriction procedure regarding organizes $(r, \theta)$. The portable hub can refresh the estimations of $(r, \theta)$ in standard interims and keep up a store. Presently, the immediate separation Ri between portable hub and APs is determined by using

$$
\begin{aligned}
& R_{i}=\sqrt{r^{2}+D^{2}-2 r D \cos \left((2 i-1) \frac{\pi}{6}-\theta\right)} \\
& \text { Where, } \quad D=\sqrt{3} R
\end{aligned}
$$

At the point when the separation between versatile hub and current AP is more prominent than a fixed edge level then the handoff procedure is started and the AP which is closest is chosen for relationship by portable hub. There is no need of handoff when the portable hub is in the in-hover of the cell while the circum-circle characterizes the scope of individual AP. This is shown in figure 2.

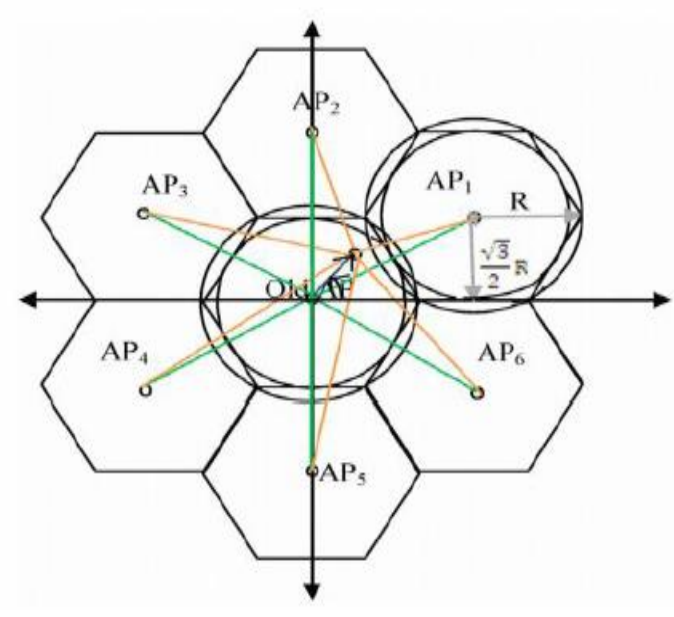

Fig 2: Distance Measurement Technique

By diminishing the quantity of channels to be examined, handoff delay in filtering stage can be decreased. Utilizing certain measures the predetermined number of channels can be chosen to examine, is known as particular channel checking. Symmetrical guideline can be utilized as the choice measures. The channels Ch1, Ch6 and Ch11 are non-covering diverts in the IEEE 802.11 range which are called symmetrical channels.

Agreeing the condition 4 in the event that $f(n)=1$; the specific channel will be filtered then just, implies $f(n)$ chooses whether channel number $\mathrm{n}$ is to be checked. Hindrance of this plan is that solitary examining of the symmetrical channels will miss the AP which has the more noteworthy quality. Pre-examining is acquainted with in to broaden specific checking. Accessible channels set apart by the cover are examined by STA before the handoff is activated. This is shown in simulation output shown in figure 3 . 


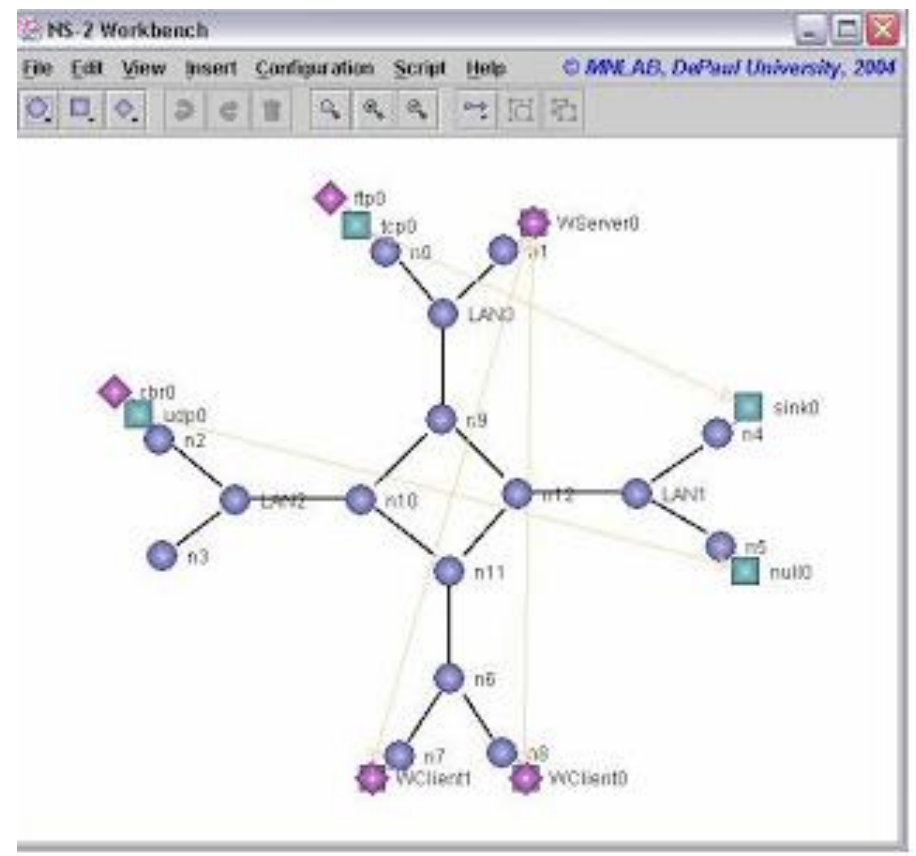

Fig 3: Simulation output showing hand-off

For our investigation, two APs are arranged at positions An and B, individually and they are isolated by separation D. A STA ventures out from AP A to AP B at a speed of CV . n STA ventures out from AP A to AP B at a speed of $\mathrm{CV}$. When the STA is situated at the separation of d from AP A, the got signal qualities from both APs, specifically ra and $\mathrm{rb}$. Leave the got signal quality alone indicated as

$$
\begin{gathered}
r a=P L 1-P L 2 \log (d)+x \\
r b=P L 1-P L 2 \log (D-d)+y
\end{gathered}
$$

Where $0 \leq \mathrm{D}$. Constants PL1 and PL2 are the parameters for way misfortune in a 802.11a WLAN condition. We consider that STA estimates the sign quality intermittently and is shown in figure 4.

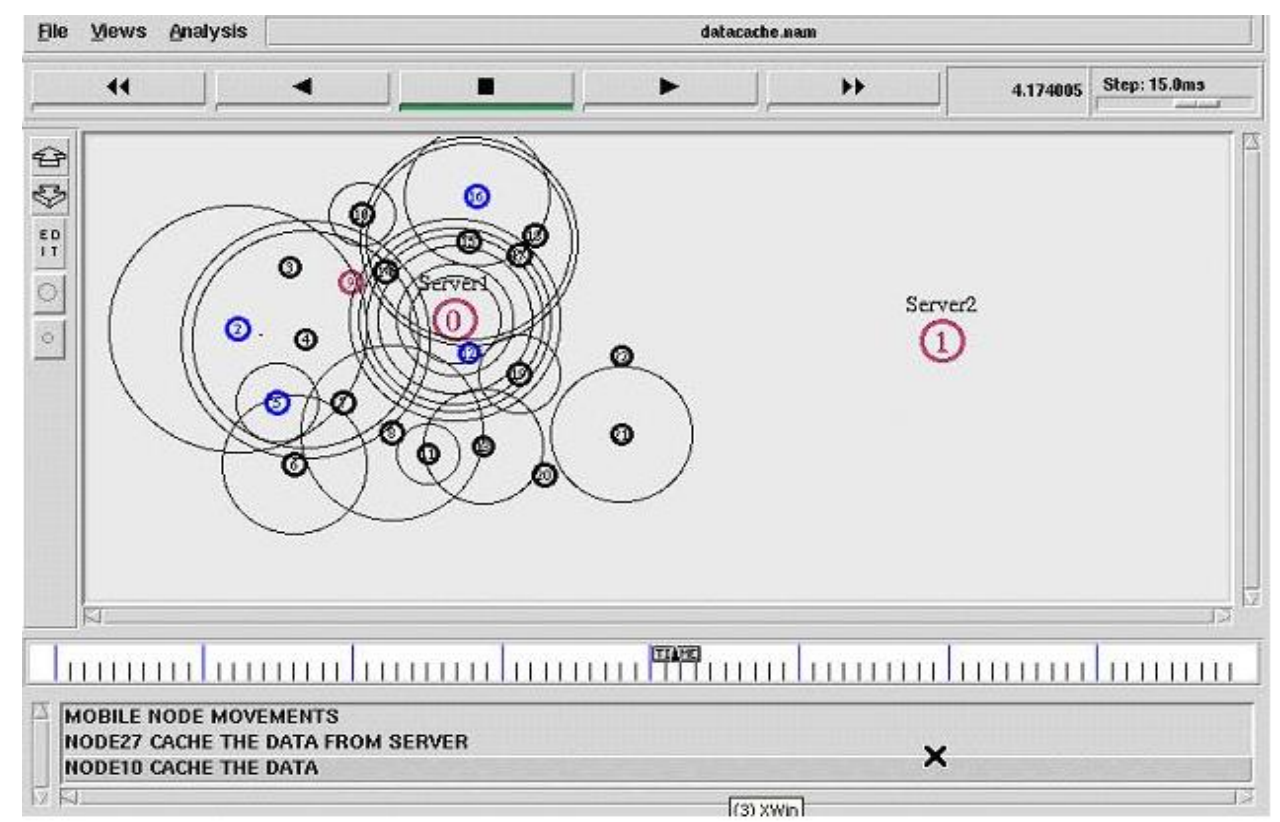

Fig 3: Simulation output showing STA estimation 
Hand off technique is pragmatic since APs communicate their reference point outlines in a time of $100 \mathrm{~ms}$. $\mathrm{x}$ and $\mathrm{y}$ are free and indistinguishably disseminated zero mean fixed guassian irregular procedure. In this investigation the 802.11 filtering starts when the got signal quality dips under edge $C \neg \neg$ h.. The checking likelihood for the ith interim is given by

$$
\mathrm{Ps}(\mathrm{i})=\mathrm{P}(\mathrm{Ch}>\mathrm{r} \mathrm{a}(\mathrm{i}))+\mathrm{P}(\mathrm{Ch}>\mathrm{r} \mathrm{b}(\mathrm{i}))
$$

If the received signal strength varies depends on average standard deviation and varience

$$
\begin{gathered}
\mathrm{P}(\mathrm{Ch}>\mathrm{r} \mathrm{a}(\mathrm{i}))=1 / 2(1+\operatorname{erf}((\mathrm{Ch}-\mathrm{r} \text { a(i) }) / \sqrt{2} \sigma) \\
\mathrm{P}(\mathrm{Ch}>\mathrm{r} \mathrm{b}(\mathrm{i}))=1 / 2(1+\operatorname{erf}((\mathrm{Ch}-\mathrm{r} \mathrm{b}(\mathrm{i})) / \sqrt{2} \sigma)
\end{gathered}
$$

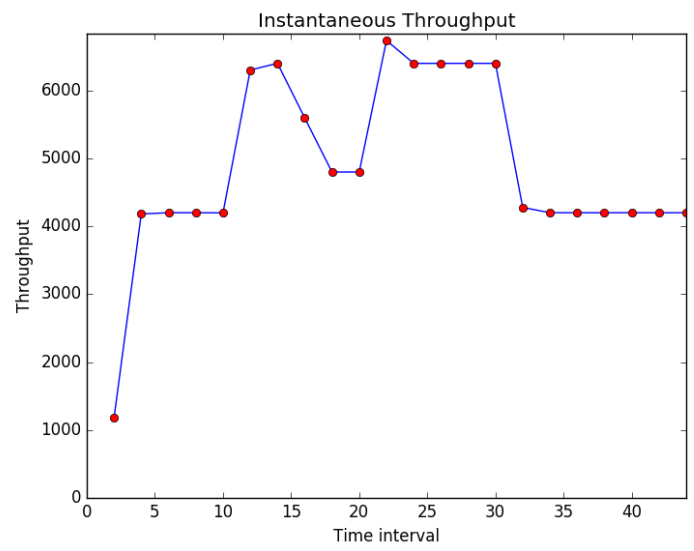

Fig 4: Simulation output showing throughput

There are two cases for STA related to an AP after ith estimation. At STA hand off to the AP when it perceive that AP gives better sign quality by filtering or it remains with the AP than since got signal more grounded than Ch.

$$
\begin{aligned}
& \mathrm{PA}(\mathrm{i})=\mathrm{Ps}(\mathrm{i}) \mathrm{P}(\mathrm{r} \mathrm{a}(\mathrm{i}))>\mathrm{r} \text { b(i) })+(1-\mathrm{P}(\mathrm{Ch}>\mathrm{r} \mathrm{a}(\mathrm{i}))) \\
& \mathrm{PB}(\mathrm{i})=\mathrm{Ps}(\mathrm{i}) \mathrm{P}(\mathrm{r} \text { b(i)) }>\mathrm{r} \mathrm{a}(\mathrm{i}))+(1-\mathrm{P}(\mathrm{Ch}>\mathrm{r} \mathrm{b}(\mathrm{i}))) \mathrm{PB}(\mathrm{i}-1) \\
& \text { Total number packet loss }=\sum_{i+0}^{\{D / C p C v\}} N \boldsymbol{W}
\end{aligned}
$$

The $\mathrm{X}$ and $\mathrm{Y}$ graphs shows proposed system packet loss is very less compared to existing system and its latency is reduced with respect to packet loss. Simulation environment is created based on pre authentication and without pre authentication scanning.

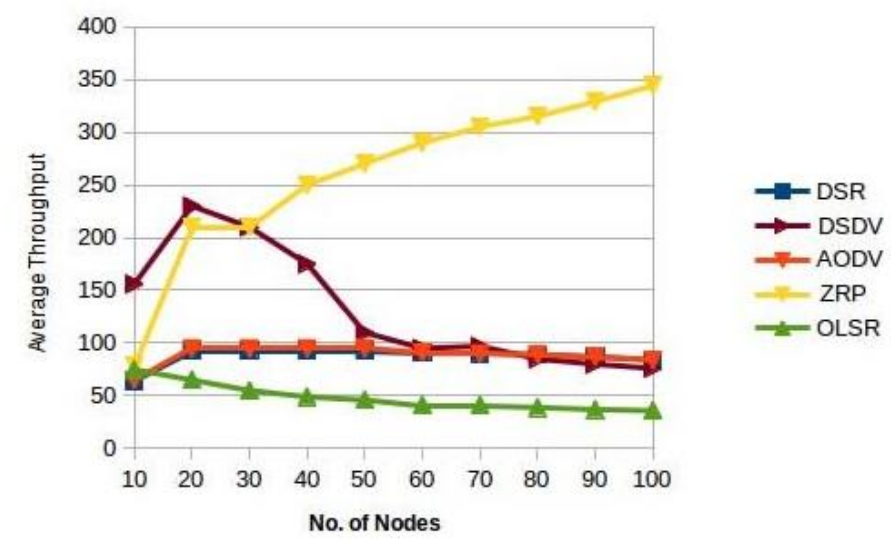

Fig 5: Simulation output showing throughput comparison 


\section{Conclusion}

We have proposed a novel scanning plan for the 802.11 handoff and the exploratory outcomes show that the proposed scanning plan extraordinarily decreases information outline misfortunes, though the scanning time stays unaltered. We have evaluated the quantity of bundle misfortunes relying upon the handoff plots by numerically examining the presentation of the handoff plans. In synopsis, the proposed scanning plan is valuable for the application, for example, voice over IP and mixed media gushing administrations since those applications are very touchy to information outline misfortunes.

\section{References}

[1] S. Jin, K. Han, and S. Choi, "A novel Idle mode operation in IEEE 802.11 WLANs: Prototype implementation and empirical evaluation," in Proc. ACM WMASH, Sep. 2006, pp. 71-80.

[2] S. Jin, K. Han, and S. Choi, "A Novel Idle Mode Operation in IEEE 802.11 WLANs," in Proc. IEEE ICC, Jun. 2006, pp. 4824-4829.

[3] S. Kim, S. Choi, S.-k. Park, J. Lee, and S. Kim, "An empirical measurements-based analysis of public WLAN handoff operations," in Proc. WILLOPAN, Jan. 2006, pp.6.

[4] S. Jin, M. Choi, L. Wang, and S. Choi, "Fast scanning schemes for IEEE 802.11 WLANs in virtual AP environments," Elsevier Computer Networks., vol. 55, no. 10, pp. 2520-2533, Jul. 2011.

[5] K. Ramachandran, S. Rangarajan, and C. J. Lin, "Make-before-break mac layer handoff in 802.11 wireless networks," in Proc. IEEE ICC, Jun.2006, pp. 4818-4823.

[6] Y.-S. Chen, M.-C. Chuang, and C.-K. Chen, "Deucescan: Deuce-based fast handoff scheme in IEEE 802.11 wireless networks," "IEEE Trans. Veh. Technol., vol. 57, no. 2, pp. 1126-1141, Mar. 2008.

[7] I. Ramani and S. Stephan, "SyncScan: Practical fast handoff for 802.11 infrastructure networks," in Proc. IEEE INFOCOM, Mar. 2005, pp. 675-684.

[8] H. Velayos and G. Karlsson, "Techniques to reduce the IEEE $802.11 \mathrm{~b}$ handoff time," in Proc. IEEE ICC, Jun. 2004, pp. 3844-3848.

[9] Y. Zheng, T. Shi, X. Xu, H. Yuan and T. Yao, "Research on WLAN planning problem based on optimization models and multi-agent algorithm," 2017 IEEE International Conference on Cybernetics and Intelligent Systems (CIS) and IEEE Conference on Robotics, Automation and Mechatronics (RAM), Ningbo, 2017, pp. 249-254, doi: 10.1109/ICCIS.2017.8274782.

[10] H. M. T. Al-Hilfi, B. A. Salih and I. Marghescu, "Design of secured WLAN by using "packet filtering firewall"," 2017 International Conference on Wireless Communications, Signal Processing and Networking (WiSPNET), Chennai, 2017, pp. 1857-1862, doi: 10.1109/WiSPNET.2017.8300083. 